\title{
In Vitro Comparison of Isometric and Stop-Test Contractility Parameters for the Urinary Bladder
}

\author{
R. van Mastrigt and J. J. Glerum \\ Department of Urology, Erasmus University Rotterdam, Rotterdam, The Netherlands
}

Accepted: May 16, 1984

Summary. Contractility parameters in the urinary bladder can be calculated from isometric contractions (no extra patient load as compared to routine cystometry) or from stop-tests (more accurate, simpler analysis). A stop-test involves a voluntarily interrupted micturition with pressure and flow measurement. In a series of measurements in vitro on pig urinary bladder strips, parameters of the first type, obtained either by analyzing isometric contractions in terms of the Hill model, or by making phase plots, were compared to parameters of the second type. A good correlation was found. The parameter correlating best with the maximal contraction velocity of the bladder, normalized for differences in initial muscle length, as obtained from stop-test, is the isometric contraction force, which can be obtained from an isometric contraction by either of the two analysis techniques. Clinically, making phase plots seems more promising than analyzing contractions in terms of the Hill model.

Key words: Contractility, Smooth muscle, Isometric, Urinary bladder, Stop-test, Force-velocity relation.

\section{Introduction}

A contractility parameter describes quantitatively the ability of a muscle to shorten and/or to develop force [8]. For the urinary bladder contractility parameters might be used to investigate pathological conditions, to evaluate the results of treatment and to predict the possibility of recovery of the detrusor muscle. Two types of contractility parameters can be distinguished, parameters determined from isometric contractions, and parameters determined from measurements made during shortening of the muscle [16]. Both spontaneous contractions during the filling phase of a routine cystometry procedure, and the pressure rise which can be recorded just before micturition starts can be considered as isometric contractions. The recording of these contractions therefore places no extra burden on the patient. The analysis is complicated however, and the accuracy may be poor [10]. A method of measuring contractility during shortening of the muscle is the stop-test [1], which consists of an interrupted micturition with pressure and flow measurement. The interruption of the stream, either mechanically, or voluntarily by the patient, introduces the risk of detrusor inhibition, but the analysis of these measurements is relatively straightforward. The present study was undertaken to investigate the correlation between the two types of contractility measurements. A good correlation would mean that it is possible to combine the patient friendliness of isometric contraction measurements with the accuracy of the stop-tests. The comparison was performed in vitro. Parameters obtained by estimating the force-velocity relation of contracting muscle from isometric contractions $[6,10]$ and parameters obtained from phase plots of isometric contractions [12] were compared to parameters calculated from an in-vitro analog of the stop-test [2]. Isometric contractions were generated by electrical stimulation of bladder muscle strips. The invitro analog of the stop-test involved recording the contraction force during mechanically controlled shortening of an electrically stimulated muscle strip, and recording of the force immediately after this shortening had been stopped.

\section{Material and Methods}

Experiments were performed on 12 strips of urinary bladder taken from pigs that were sacrificed for cardiac research. From each bladder a strip of about $15 \times 23 \mathrm{~mm}$ was excised. The strips were mounted vertically between two clamps with penetrating pins. The lower clamp was fixed to the bottom of a container filled with warmed and aerated Krebs solution [12]. The upper clamp was connected to a Grass force transducer which could be moved at a constant, preset velocity by means of an erect Harvard infusion pump. The signal from the force transducer was amplified and fed into a Texas Instruments minicomputer via an $\mathrm{A} / \mathrm{D}$ converter. Initial 


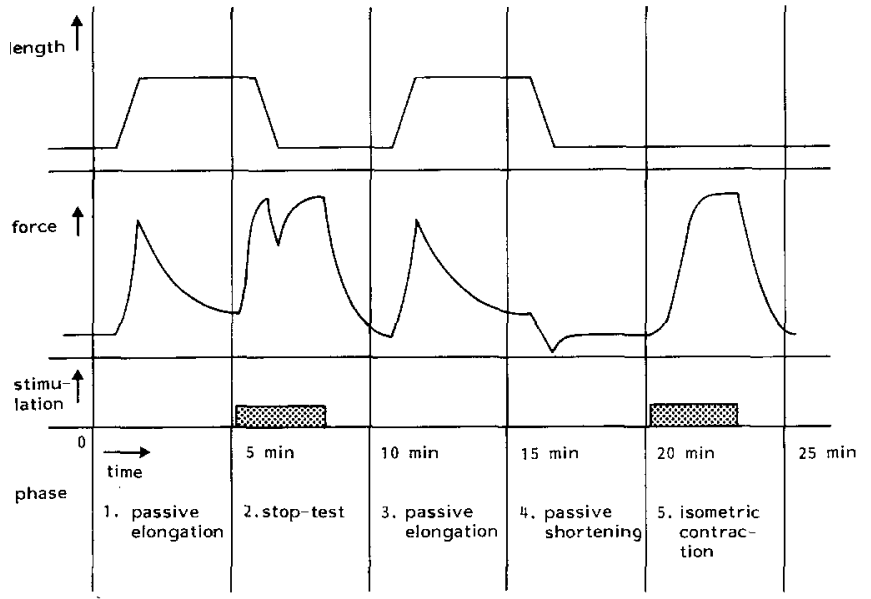

Fig. 1. Sequence of events in one measurement, comprising five phases. Time axis not drawn to scale. Stimulation lasts for $18 \mathrm{~s}$ in a $5 \mathrm{~min}$ phase, shortening or elongation takes place from 8 th to 10 th $\mathrm{s}$ of a phase

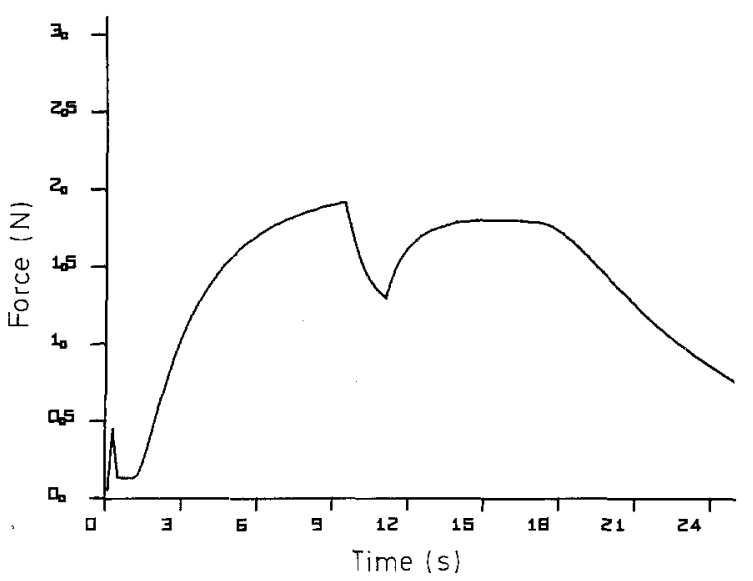

Fig. 2. Force as a function of time for a stop-test measurement. Shortening speed was set to $0.316 \mathrm{~mm} / \mathrm{s}$

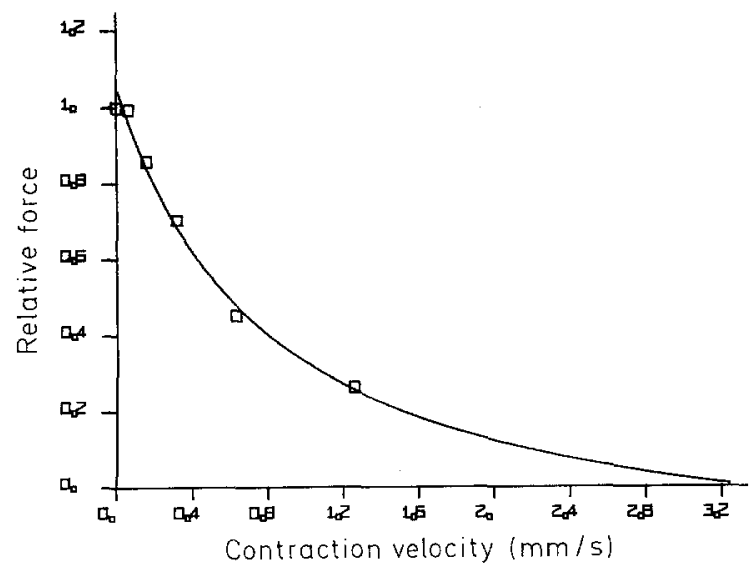

Fig. 3. Relative force-velocity curve. Squares represent results of five stop-tests, curve represents fitted hyperbolic function length of the strips was set by straining them at a rate of $0.0632 \mathrm{~mm} / \mathrm{s}$ until a peak force of $50 \mathrm{gf}$ was measured. Strips were made to contract by applying alternating voltage pulses to two platinum field electrodes of size $15 \times 20 \mathrm{~mm}$, placed on either side of the strip. Unless stated otherwise, $20 \mathrm{~V}, 100 \mathrm{~Hz}, 5 \mathrm{~ms}$ pulses were applied. Both the movement of the force-transducer and the switching on and off of the stimulation voltage were controlled by the computer. On each strip two series of measurements were performed. In three strips the second series of measurements was identical to the first series. In five strips, the length of the strip was changed before the second series, in two strips stimulation voltage was reduced and in two strips the stimulation frequency was reduced. Each series consisted of five measurements, made at five different speed settings of the pump, in the following order: $0.0632 \mathrm{~mm} / \mathrm{s}-0.158 \mathrm{~mm} / \mathrm{s}-$ $1.26 \mathrm{~mm} / \mathrm{s}-0.316 \mathrm{~mm} / \mathrm{s}-0.63 \mathrm{~mm} / \mathrm{s}$. Each measurement comprised the following five phases, each phase lasting $5 \mathrm{~min}$ (Fig. 1).

1. Length of the strip was increased by letting the pump run for exactly $2 \mathrm{~s}$. The increase in length therefore depended on the speed setting of the pump. The strip was allowed to relax for $5 \mathrm{~min}$ at this increased length [4].

2. The strip was stimulated to contract for $18 \mathrm{~s}$; between the 8 th and 10th $\mathrm{s}$ it was allowed to shorten by the infusion pump, so as to end at the initial length. This phase formed the actual stop-test $[2,5]$.

3. Passive elongation of strip as in phase 1 .

4. Shortening of the strip exactly as in phase 2 , but without electrical stimulation, so that the passive response of the strip to the shortening could be measured and subtracted from the total response measured in phase 2 , so as to yield the active response only.

5. Isometric contraction of the strip, brought about by electrical stimulation for $18 \mathrm{~s}$.

\section{Data Processing Methods}

I. Stop-Tests. Figure 2 shows an example of a stop-test. These were processed in the following way. From the minimum force in the "dip" where shortening was stopped, the passive force at the corresponding time in the passive shortening measurement (phase 4 , Fig. 1) was subtracted. The resulting active force during shortening was divided by the maximum force reached after the dip in Fig. 2, minus the force at the corresponding point in phase 4 . This yielded a relative force at a given shortening velocity and a given muscle length [2], corrected for passive force changes. Five such points, measured at five different shortening velocities, yielded a relative force-velocity curve (Fig. 3). This curve was fitted with a hyperbolic function [7] according to Hill [3], with fixed curvature $(\mathrm{a} / \mathrm{F} 0=0.25)$. This yielded the maximum contraction velocity $\mathrm{V}_{\max }$, the intersection of the curve with the velocity axis. Although in estimating $V_{\max }$ the forces were expressed relatively, in order to eliminate any effect of spontaneous differences in active force, the average maximum active force reached is also a parameter, so that one series of five measurements yielded two parameters, $V_{\max }$ and $\mathrm{F}_{\max }$

II. Isometric Contractions, Calculation of Force-Velocity Relation. Figure 4 shows an isometric contraction. Assuming the validity of the Hill equation, and a fixed series elasticity of the following form:

$\mathrm{F}=\mathrm{G} \cdot \mathrm{L} \cdot \exp (\mathrm{B} \cdot \mathrm{L})$

where $F=$ force exerted by series elastic element; $G=$ coefficient, chosen as $50 \mathrm{~N} / \mathrm{m}$; $\mathrm{L}=$ extension of series elastic element; $\mathrm{B}=\mathrm{ex}-$ ponent, chosen as $500 / \mathrm{m}$ [5] an estimate of the force-velocity relation was calculated $[10]$ and again fitted with a hyperbolic curve 


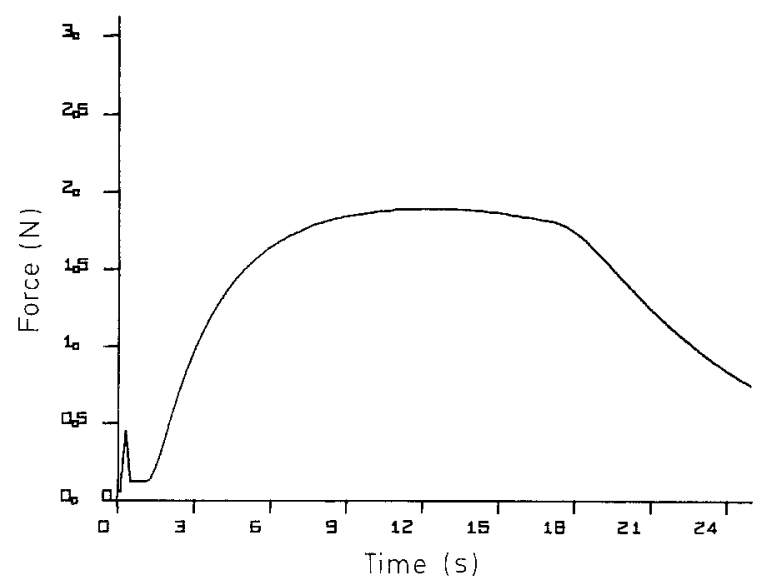

Fig. 4. Force as a function of time for an isometric contraction

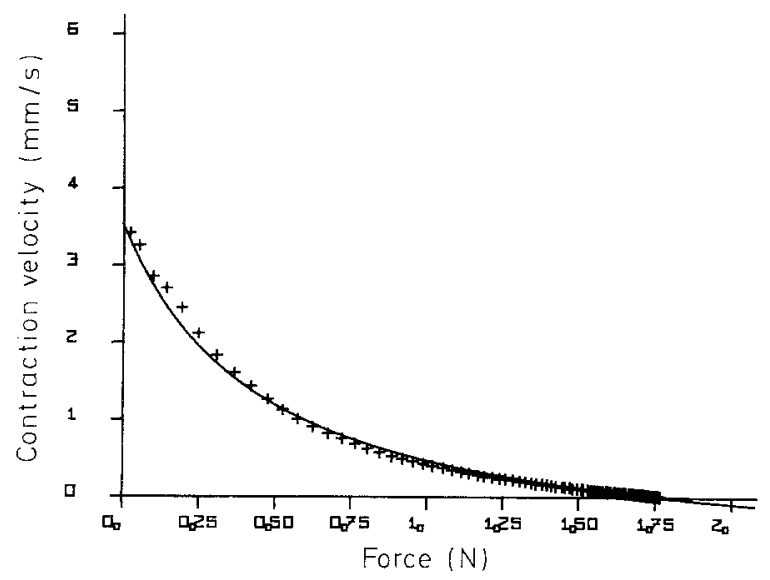

Fig. 5. Force-velocity relation (crosses) calculated from the isometric contraction shown in Fig. 4 and fitted hyperbolic function

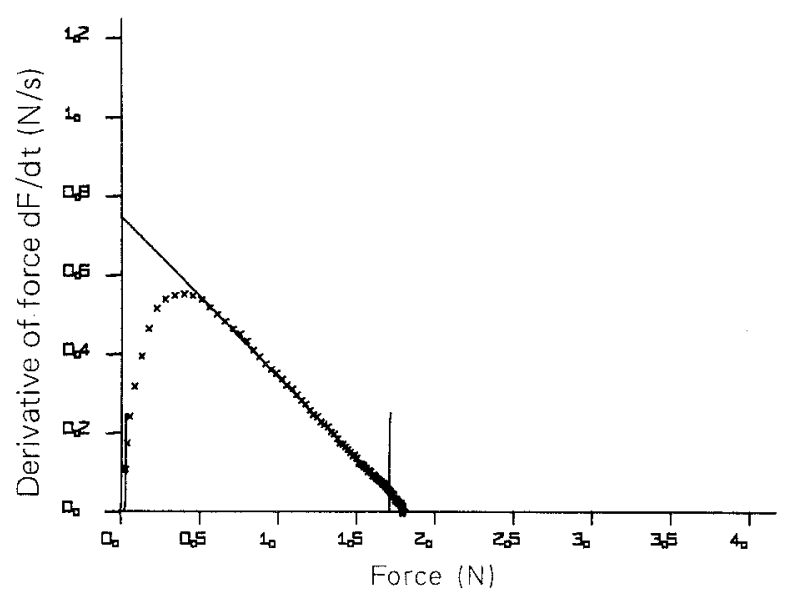

Fig. 6. Phase plot (time derivative of force as a function of force) calculated from the isometric contraction shown in Fig. 4, and fitted straight line. Vertical lines indicate beginning and end of stimulation

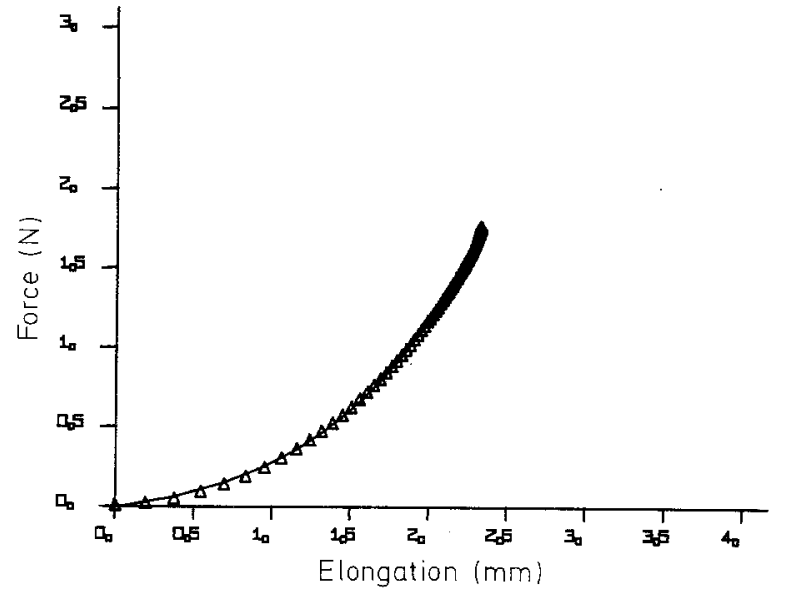

Fig. 7. Series elasticity of Hill model as calculated from the isometric contraction shown in Fig. 4 and associated stop-test (triangles). Data was fitted with function (1) (curve), see text

with fixed curvature $(\mathrm{a} / \mathrm{F} 0=0.25)$ (Fig. 5). The resulting two parameters, the intersections of the fitted curve with the velocity and force axes, were called $V_{\max }$ fit and $F_{\max }$ fit respectively, and were averaged for the five contractions in each measurement series.

III. Isometric Contractions, Calculation of Phase Plot. From the same isometric contractions a phase plot (a plot of the rate of change of force as a function of that force) was calculated [12] as shown in Fig. 6 and a straight line was fitted to the data [13]. This yielded as parameters $U$, the intersection of the fitted line with the vertical axis, and Fiso, the intersection with the horizontal axis. The parameters $U$ and Fiso were averaged for the five isometric contractions in each measurement series.

IV. Calculation of Series Elasticity. From each isometric contraction, and the corresponding stop-test an estimate of the force length relation of the series elastic component in the Hill model was calculated [5]. To this curve the function (1) was fitted. Figure 7 shows a calculated elastic characteristic and the fitted function. This analysis was applied only to the four measurements at the highest shortening velocities from each series. The resulting parameters $G$ and $B$ were averaged for the four measurements. (Another function was also fitted to the force length relation, see Appendix).

The associations among all the resulting parameters were examined by calculating Spearman's rank correlation coefficients, both for all measurements and for the first and second measurement series separately.

\section{Results}

Table 1 shows averages and standard deviations of the parameters for the first and second series of measurements separately. Since the three parameters associated with maximal force $\left(F_{\max }\right.$ from the stop-tests, $F_{\text {max fit }}$ from the force-velocity relations calculated from isometric contractions, and Fiso from the phase plots) were almost identical (correlation coefficients in the order of 0.98 ), only one is shown. In every strip all the parameters except $B$ were lower in the second measurement series than in the first, indicating a significant deterioration of the preparation. This effect completely masked any changes due to length 


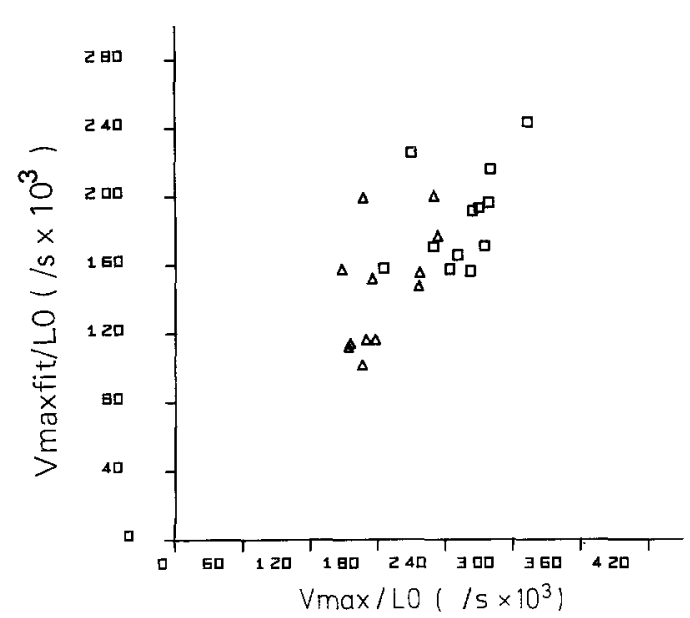

Fig. 8. Scatter plot showing relation between the parameters $\mathrm{V}_{\max }$ fit $/ \mathrm{LO}$ and $\mathrm{V}_{\max } / \mathrm{LO}$. Squares indicate first measurement series, triangles second measurement series made on each strip

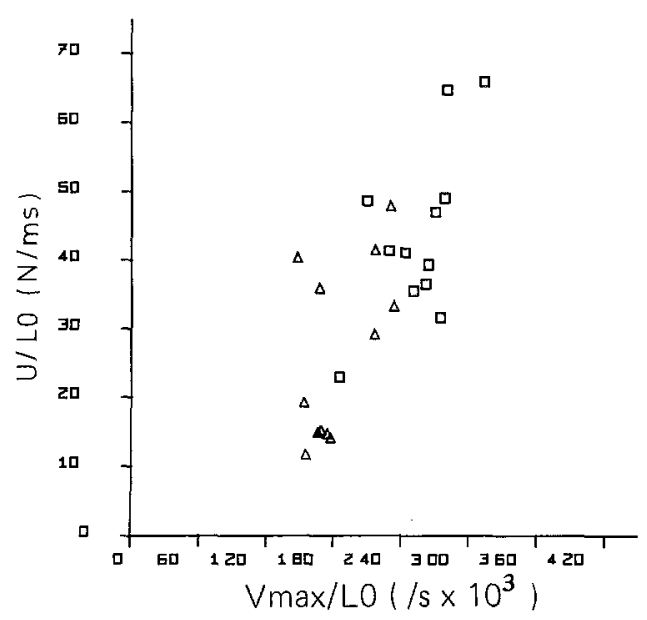

Fig. 9. Scatter plot showing relation between the parameters $\mathrm{U} / \mathrm{LO}$ and $V_{\max } / L 0$. Squares indicate first measurement series, triangles second measurement series made on each strip

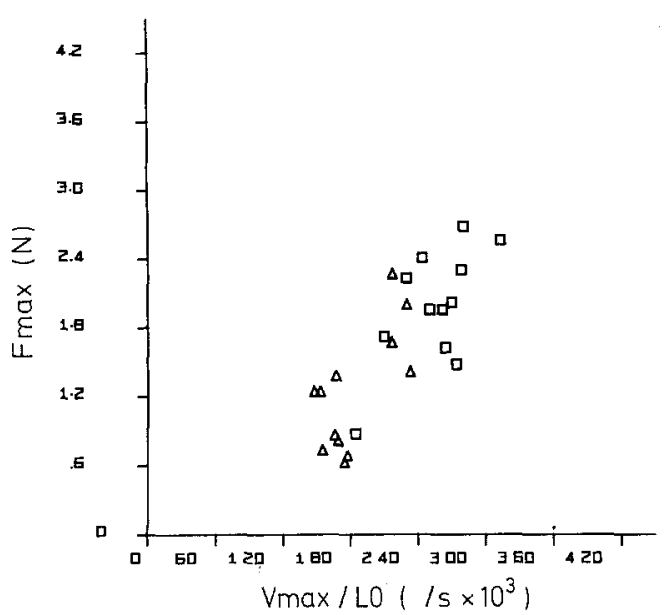

Fig. 10. Scatter plot showing relation between the parameters $F_{\max }$ and $\mathrm{V}_{\max } /$ LO. Squares indicate first measurement series, triangles second measurement series made on each strip

or different stimulus conditions in the second series. Since the velocity parameters $V_{\max }, V_{\max \text { fit }}$ and perhaps also $U$ may be expected to be linearly dependent on the initial length of the strip (a larger length means more cells in series, the shortening velocities of the cells being added) the quotient of these parameters and initial length is also displayed. There is a systematic difference between $V_{\max }$ and $V_{\max f i t}$, which is due to the fact that the series elasticity of these strips is significantly stiffer than that assumed (first measurement series average $G=108 \mathrm{~N} / \mathrm{m}$ whereas assumed value was $G=50 \mathrm{~N} / \mathrm{m}$ ). Table 2 shows the dependence of the parameters on initial length. It can be seen that both $\mathrm{V}_{\max }$ and $\mathrm{V}_{\max \text { fit }}$ indeed depend significantly on $\mathrm{L} 0$, while the normalized parameters $V_{\max } / \mathrm{LO}$ and $\mathrm{V}_{\max \text { fit }} / \mathrm{LO}$ do not do so. For the parameter U the significance of the dependence is borderline. Table 3 shows the correlations between isometric and shortening contractility parameters. The very strong correlation between $\mathrm{V}_{\max }$ and $\mathrm{V}_{\text {max fit }}$ is partly due to their mutual dependence on $\mathrm{LO}$,

Table 1. Averages and standard deviations of the parameters for the first and second series of measurements on each urinary bladder strip. Measurements from 12 strips

\begin{tabular}{|c|c|c|c|c|c|}
\hline \multirow[t]{2}{*}{ Parameter } & \multicolumn{2}{|c|}{ First series } & \multicolumn{2}{|c|}{ Second series } & \\
\hline & Average & Standard dev. & Average & Standard dev. & \\
\hline$F_{\max }$ & 1.98 & 0.48 & 1.25 & 0.51 & $(\mathrm{~N})$ \\
\hline $\mathrm{V}_{\max }$ & 6.06 & 1.54 & 4.40 & 1.37 & $(\mathrm{~mm} / \mathrm{s})$ \\
\hline $\mathrm{V}_{\max } / \mathrm{LO}$ & 0.255 & 0.033 & 0.184 & 0.030 & $(/ \mathrm{s})$ \\
\hline $\mathrm{V}_{\max f i t}$ & 4.34 & 0.700 & 3.43 & 1.00 & $(\mathrm{~mm} / \mathrm{s})$ \\
\hline $\mathrm{V}_{\max f i t} / \mathrm{LO}$ & 0.187 & 0.028 & 0.146 & 0.033 & $(/ \mathrm{s})$ \\
\hline $\mathrm{U}$ & 1.02 & 0.27 & 0.641 & 0.36 & $(\mathrm{~N} / \mathrm{s})$ \\
\hline $\mathrm{U} / \mathrm{L} 0$ & 43.7 & 12.0 & 26.5 & 12.4 & $(\mathrm{~N} / \mathrm{ms})$ \\
\hline G & 108 & 45 & 82 & 62 & $(\mathrm{~N} / \mathrm{m})$ \\
\hline B & 495 & 125 & 627 & 266 & $(/ \mathrm{m})$ \\
\hline
\end{tabular}


Table 2. Correlations between contractility parameters and the initial length of the muscle strip. Spearman's rank correlation coefficients calculated both for all measurements (first line for each parameter, $n=24$ ) and for the first and second measurement series separately (second line, $n=12$ ). Significance of correlation coefficients at the $1 \%$ level is indicated by an $*$ The critical value of Spearman's rank correlation coefficient at the $1 \%$ level is 0.485 for $n=24$ and 0.703 for $n=12$

\begin{tabular}{ll}
\hline Parameter & L0 \\
\hline Correlation with: & \\
$\mathrm{F}_{\max }$ & 0.34 \\
& $0.14 / 0.45$ \\
$\mathrm{~V}_{\max }$ & $0.82^{*}$ \\
& $0.90 / 0.92^{* *}$ \\
$\mathrm{~V}_{\max } / \mathrm{LO}$ & 0.20 \\
& $-0.03 / 0.20$ \\
$\mathrm{~V}_{\max }$ & $0.65^{*}$ \\
& $0.82 / 0.59^{*}$ \\
$\mathrm{~V}_{\max }$ fit $/ \mathrm{LO}$ & -0.29 \\
$\mathrm{U}$ & $-0.64 /-0.20$ \\
& $0.49^{*}$ \\
$\mathrm{U} / \mathrm{LO}$ & $0.49 / 0.52$ \\
& 0.05 \\
& $-0.28 / 0.24$
\end{tabular}

Table 3. Correlations between isometric and shortening contractility parameters. Spearman's rank correlation coefficients calculated both for all measurements (first line for each parameter, $n=24$ ) and for the first and second measurement series separately (second line, $n=12$ ). Significance of correlation coefficients at the $1 \%$ level is indicated by an *. The critical value of Spearman's rank correlation at the $1 \%$ level is 0.485 for $n=24$ and 0.703 for $n=12$

\begin{tabular}{|c|c|c|c|}
\hline Parameter & $\mathrm{F}_{\max }$ & $\mathrm{V}_{\max }$ & $\mathrm{V}_{\max } / \mathrm{L} 0$ \\
\hline \multicolumn{4}{|c|}{ Correlation with: } \\
\hline $\mathrm{F}_{\max }$ & $\begin{array}{l}x \\
x\end{array}$ & $\begin{array}{l}0.70^{*} \\
0.21 / 0.58\end{array}$ & $\begin{array}{l}0.77^{*} \\
0.53 / 0.47\end{array}$ \\
\hline$V_{\max }$ & $\begin{array}{l}0.70^{*} \\
0.21 / 0.58\end{array}$ & $\begin{array}{l}x \\
x\end{array}$ & $\begin{array}{l}x \\
x\end{array}$ \\
\hline $\mathrm{V}_{\max } / \mathrm{L} 0$ & $\begin{array}{l}0.77^{*} \\
0.53 / 0.47\end{array}$ & $\begin{array}{l}x \\
x\end{array}$ & $\begin{array}{l}x \\
x\end{array}$ \\
\hline$V_{\max f i t}$ & $\begin{array}{l}0.83^{*} \\
0.45 / 0.83^{*}\end{array}$ & $\begin{array}{l}0.89^{*} \\
0.79 / 0.73^{*} *\end{array}$ & $\begin{array}{l}x \\
x\end{array}$ \\
\hline $\mathrm{V}_{\max f i t} / \mathrm{LO}$ & $\begin{array}{l}0.63 * \\
0.34 / 0.57\end{array}$ & $\begin{array}{l}x \\
x\end{array}$ & $\begin{array}{l}0.65^{*} \\
0.55 / 0.51\end{array}$ \\
\hline $\mathrm{U}$ & $\begin{array}{l}0.93^{*} \\
0.83 / 0.94^{* *}\end{array}$ & $\begin{array}{l}0.79^{*} \\
0.58 / 0.63\end{array}$ & $\begin{array}{l}x \\
x\end{array}$ \\
\hline $\mathrm{U} / \mathrm{L} 0$ & $\begin{array}{l}0.90 * \\
0.80 / 0.89 * *\end{array}$ & $\begin{array}{l}x \\
x\end{array}$ & $\begin{array}{l}0.65^{*} \\
0.55 / 0.33\end{array}$ \\
\hline
\end{tabular}

Table 4. Correlations between the parameters describing the series elasticity and initial muscle length and stop-test parameters. Spearman's rank correlation coefficients calculated both for all measurements (first line for each parameter, $n=24$ ) and for the first and second measurement series separately (second line, $n=12$ ). Significance of correlation coefficients at the $1 \%$ level is indicated by an *. The critical value of Spearman's rank correlation coefficient at the $1 \%$ level is 0.485 for $n=24$ and 0.703 for $n=12$

\begin{tabular}{|c|c|c|c|c|}
\hline Parameter & Lo & $F_{\max }$ & $\mathrm{V}_{\max }$ & $\mathrm{V}_{\max } / \mathrm{L} 0$ \\
\hline \multicolumn{5}{|c|}{ Correlation with: } \\
\hline $\mathrm{G}$ & $\begin{array}{l}0.27 \\
0.06 / 0.39\end{array}$ & $\begin{array}{l}0.69 * \\
0.51 / 0.69\end{array}$ & $\begin{array}{l}0.54^{*} \\
0.24 / 0.47\end{array}$ & $\begin{array}{l}0.49^{*} \\
0.41 / 0.29\end{array}$ \\
\hline B & $\begin{array}{l}-0.40 \\
-0.37 /-0.48\end{array}$ & $\begin{array}{l}-0.37 \\
-0.09 /-0.41\end{array}$ & $\begin{array}{l}-0.56^{*} \\
-0.54 /-0.51\end{array}$ & $\begin{array}{l}-0.46 \\
-0.47 /-0.31\end{array}$ \\
\hline
\end{tabular}

since the correlation between $\mathrm{V}_{\max } / \mathrm{LO}$ and $\mathrm{V}_{\max \text { fit }} / \mathrm{LO}$ is weaker, although still significant. The correlation of $\mathrm{V}_{\max } / \mathrm{LO}$ with $\mathrm{U} / \mathrm{LO}$ is as good as that with $\mathrm{V}_{\max \text { fit }} / \mathrm{LO}$. There is a very strong correlation between $F_{\max }$ and the velocity parameters, especially with U. Figs. 8,9 and 10 show scatter plots of the parameters $V_{\text {max fit }} / \mathrm{LO}, \mathrm{U} / \mathrm{LO}$ and $F_{\max }$ as functions of $\mathrm{V}_{\max } / \mathrm{LO}$ and indicate that the correlations obtained do not depend on one or two extreme data points. Table 4 shows correlations between the parameters describing the series elasticity and initial muscle length and stop-test parameters. It can be seen that the series elasticity parameters are not correlated with initial length. Although they are correlated with $V_{\max }$ only the parameter $\mathrm{G}$ is correlated with the normalized parameter $\mathrm{V}_{\max } / \mathrm{LO}$.

\section{Discussion}

The object of this study was to investigate whether contractility parameters determined from isometric contractions, which make little or no extra demand from the patient as compared to standard cystometry procedures, can replace parameters determined from stop-tests, which are more accurate but make a (voluntarily) interrupted flow measurement necessary. This investigation was performed in vitro. Contractility parameters determined from an in vitro analog of the stop-test were compared to parameters determined from isometric contractions of pig urinary bladder strips. Although the bladder strip preparations deteriorated rapidly, because of repeated stimulation for long periods and repeated straining by up to $30 \%$, the average 
maximum stress $(34,000 \mathrm{~N} / \mathrm{m} 2)$, the value of $\mathrm{V}_{\max } / \mathrm{L} 0$ (about $0.25 / \mathrm{s}$ ) and the series elasticity (extension at isometric force about $10 \% \mathrm{~L} 0$ ) are comparable with the values reported for other smooth muscles [14] and also with those found for the rabbit urinary bladder [15] (apart from series elasticity). Theoretically the velocity parameters may be expected to be linearly dependent on initial muscle length, whereas the force parameters should be independent of it. This is confirmed in Table 1 . Since the force parameter obtained from stop-tests is identical to the comparable parameter, obtained from isometric contractions, we need only discuss the velocity parameters here. It was found that the assumed series elasticity function (1), which was used to calculate force-velocity relations from isometric contractions, fits the data well, but that the assumed values of the parameters did not match those actually found. This resulted in a value for $V_{\text {max fit }}$ which was systematically too low. This can easily be changed in further measurements. The basic assumption is that the series elasticity is constant. Although no correlation exists with initial length, both series elasticity parameters are correlated with $V_{\max }$ and the parameter $G$ is correlated with $\mathrm{V}_{\max } / \mathrm{L} 0$. The correlation of this parameter with $\mathrm{V}_{\max \text { fit }} / \mathrm{L} 0$ is significantly stronger, however, so that the largest part of the variability in $V_{\max } / \mathrm{L} 0$ can be attributed to $\mathrm{V}_{\max \text { fit }} / \mathrm{L} 0$ and not to the series elasticity.

Our study shows that contractility parameters derived from stop-tests and those derived by a force-velocity anal$y$ sis of isometric contractions give comparable information. One problem however is that it is often impossible to fit the force-velocity relations derived from clinical isometric contractions with a hyperbolic Hill equation [10]. Since the correlation of the parameter $\mathrm{V}_{\max } / \mathrm{L} 0$ with $\mathrm{U} / \mathrm{LO}$ is as good as that with $V_{\max }$ fit $/ \mathrm{LO}$, an alternative is to apply the phase plot analysis to clinical data. This analysis has the advantage of involving no complicated assumptions, but the disadvantage that it yields a parameter $U$ which is theoretically not directly related to $\mathrm{V}_{\max }$. Nevertheless $\mathrm{U}$ is strongly correlated with $V_{\max }$, and has also been shown to be well correlated with contractility parameters from clinical pressure-flow plots [11]. The parameter U/LO is strongly correlated with $\mathrm{F}_{\max }$, and $\mathrm{F}_{\max }$ is also the parameter which correlates best with $\mathrm{V}_{\max } / \mathrm{L} 0$. These correlations should be interpreted carefully. The correlation of $U$ or U/L0 with $F_{\max }$ can easily be understood from the phase plot analysis, in which a straight line is fitted to the isometric contraction data (see Fig. 6) which usually has a constant slope [12]. The correlation between $V_{\max }$ and $F_{\max }$ has been found before [10]. In the present series of measurements, a distinction should be made between correlations between parameters measured on different strips, and correlations between parameters measured on one strip. For example, if the association between $V_{\max }$ and $L 0$ is examined either for the first or for the second series of measurements then one obtains higher correlation coefficients that if the entire set of measurements is used, as shown in Table 2, since LO has the same value in both the first and the second measurement. On the other hand, if one is calculating a correlation coefficient for the whole series, an exaggerated value may be obtained because of the deterioration of the strip, which tends to decrease all parameters simultaneously. For the parameters $\mathrm{V}_{\max } / \mathrm{LO}$ and $F_{\max }$ the correlation coefficients for the first and second measurement series separately show that the overall correlation coefficient is indeed exaggerated in this way. This can also be seen in Fig. 10, in which groups of triangles and squares are rather far apart. This effect is less pronounced in Fig. 8, as is the decrease in the corresponding correlation coefficients in Table 2. From this in vitro study we conclude that contractility parameters determined from isometric contractions can replace stop-test parameters. In the clinic the phase plot approach is advisable. Not only is a phase plot probably the simplest and most reliable way of obtaining a good estimate of the important parameter $F_{\max }$ (in case of clinical isometric contractions flow usually starts before $F_{\max }$ is reached, so that extrapolation is necessary to estimate this parameter), but also the parameter $U$ is obtained which is related to $V_{\max }$. Both $U$ and $V_{\max }$ should be normalized with respect to initial length of the muscle.

\section{Appendix}

From each isometric contraction, and the corresponding stop-test an estimate of the series elasticity in the Hill model was calculated [5]. To these curves two functions were fitted, the function described above (1), and the following according to [9]:

$F^{\prime}=\mathrm{G} \cdot(\exp (\mathrm{B} \cdot \mathrm{L})-1)$

A large difference existed between the fitting quality of equations (1) and (2). On average the sum of least squares associated with equation (1) was a factor 23 lower than that associated with equation (2) so that equation (1) fitted the series elasticity significantly better, and only the parameters from this equation are shown.

\section{References}

1. Griffiths DJ (1977) Urodynamic assessment of bladder function. Br J Urol 49:29-36

2. Griffiths DJ, Mastrigt R van, Duyl WA van, Coolsaet BLRA (1979) Active mechanical properties of the smooth muscle of the urinary bladder. Med Biol Eng Comp 17:281-290

3. Hill AV (1938) The heat of shortening and the dynamic constants of muscle. Proc R Soc London B 126:136-195

4. Mastrigt $\mathrm{R}$ van, Coolsaet BLRA, Duyl WA van (1978) Passive properties of the urinary bladder in the collection phase. Med Biol Eng Comp 16:471-482

5. Mastrigt R van, Griffiths DJ (1979) The contractility of the urinary bladder. Urol Int 34:410-420

6. Mastrigt $R$ van (1980) The determination of contractile properties of the urinary bladder from isometric contractions. A pilot study. Urol Res 8:237

7. Mastrigt $R$ van (1980) Fitting the Hill equation to experimental data. IEEE Trans Biomed Eng 27:413-416 
8. Mastrigt R van, Duyl WA van (1982) Mechanics of detrusor contraction. Determination of contractility. In: A course on principles of bladder function and urodynamics. Lameris, Utrecht, pp 31-36

9. Mastrigt R van, Tauecchio EA (1982) Series elastic properties of strips of smooth muscle from pig urinary bladder. Med Biol Eng Comp 20:585-594

10. Mastrigt $R$ van (1983) Determination of the contractility of children's bladders from isometric contractions. Urol Int 38 : $354-362$

11. Mastrigt $R$ van (1983) A comparison of contractility parameters determined from pressure-flow studies and isometric contractions. Proc 2nd Joint Meeting ICS/UDS, Aachen, pp 41-43

12. Mastrigt R van, Glerum JJ (1984) Electrical stimulation of smooth muscle strips from the urinary bladder of the pig. (Submitted for publication)

13. Meiss RA (1975) Graded activation in rabbit mesotubarium smooth muscle. Am J Physiol 229:455-465
14. Murphy RA (1976) Contractile system function in mammalian smooth muscle. Blood Vessels 13:1-23

15. Uvelius B (1979) Shortening velocity, active force and homogeneity of contraction during electrically evoked twitches in smooth muscle from rabbit urinary bladder. Acta Physiol Scand 106:481-486

16. Welkowitz W (1981) Indices of cardiac status. IEEE Trans Biomed Eng 28:553-567

R. van Mastrigt

Department of Urology

Erasmus University

P.O. Box 1730

NL-3000 DR Rotterdam

The Netherlands 